\title{
Application of a New Method for Analyzing Images: Two-Dimensional Non-Linear Additive Decomposition
}

MA Zaccaria, DM Drudnoy, JE Stasenko

This report was prepared as an account of work sponsored by the United States Government. Neither the United States, nor the United States Department of Energy, nor any of their employees, nor any of their contractors, subcontractors, or their employees, makes any warranty, express or implied, or assumes any legal liability or responsibility for the accuracy, completeness or usefulness of any information, apparatus, product or process disclosed, or represents that its use would not infringe privately owned rights. 


\title{
Application of a New Method for Analyzing Images: Two-Dimensional Non-Linear Additive Decomposition
}

\author{
Michael A. Zaccaria, David M. Brudnoy, John E. Stasenko \\ Lockheed Martin Company \\ P.O. Box 7450 \\ Schenectady, NY 12301-7450
}

\begin{abstract}
This paper documents the application of a new image processing algorithm, two-dimensional non-linear additive decomposition (NLAD), which is used to identify regions in a digital image whose gray-scale (or color) intensity is different than the surrounding background. Standard image segmentation algorithms exist that allow users to segment images based on gray-scale intensity and/or shape. However, these processing techniques do not adequately account for the image noise and lighting variation that typically occurs across an image. NLAD is designed to separate image noise and background from artifacts thereby providing the ability to consistently evaluate images. The decomposition techniques used in this algorithm are based on the concepts of mathematical morphology. NLAD emulates the human capability of visually separating an image into different levels of resolution components, denoted as 'coarse', 'fine', and 'intermediate.' Very little resolution information overlaps any two of the component images. This method can easily determine and/or remove trends and noise from an image. NLAD has several additional advantages over conventional image processing algorithms, including no need for a transformation from one space to another, such as is done with Fourier transforms, and since only finite summations are required, the calculational effort is neither extensive nor complicated.
\end{abstract}

Keywords: image processing, mathematical morphology, algorithm

\section{INTRODUCTION}

Mathematical morphology is a useful tool to extract features, identify defects, and reduce noise in digital images. It was originally developed by Serra ${ }^{1}$ and Matheron ${ }^{2}$ and is used to process images based on shape. Mathematical morphology can be used to describe objects by emphasizing their shapes, textures, luminosity, color and volume at each point ${ }^{3}$. It typically simplifies image data while preserving their basic shape characteristics and eliminating unrelated data ${ }^{4}$. Mathematical morphology was originally used for binary images, but was later extended to gray-scale images. In binary images, mathematical morphology alters an object's boundaries while in gray-scale images, mathematical morphology changes the shape of the intensity surface.

The non-morphological approach to image processing is based on the concept of the point-spread function and linear transforms such as convolution, the Fourier transform and wavelets ${ }^{5}$. These tend to be more complex than morphological methods and also require a greater calculational effort.

The basic operations of mathematical morphology are erosion and dilation. All other morphological operations are combinations of these two. These operations use a structuring element, which is an image that is simpler than the image being processed, to interact with the image and extract information. Different types of information can be extracted from the image by varying the structuring element.

The image processing technique discussed in this paper is the 2-D Non-Linear Additive Decomposition (NLAD) algorithm developed by Brudnoy ${ }^{6}$. This image processing algorithm is used to identify regions in a digital image whose gray-scale (or color) intensity is different than the surrounding background. These regions manifest themselves as regions of subtle intensity variations in digital images and vary in size and shape with poorly defined boundaries. Standard image segmentation algorithms exist that allow users to segment images based on gray-scale intensity and/or shape. However, these processing techniques do not adequately account for the image noise and lighting variation that 
typically occurs across an image. This algorithm decomposes an image into four separate component images; a baseline image, a noise image, a peaks image and a troughs image. This decomposition is based on resolution, and is performed in such a way that very little resolution information overlaps any two of the components.

\section{ALGORITHM DESCRIPTION}

The decomposition techniques used in NLAD are based on the concepts of mathematical morphology. NLAD emulates human vision, called resolution decomposition, in which a signal is decomposed into three distinct components, based on "coarse" (baseline), "fine" (noise), and "intermediate" (peaks/troughs) levels of visual resolution. The basic concepts are described below using 1-D NLAD. A signal, $\{\mathrm{S}[\mathrm{n}], \mathrm{n}=0,1, \ldots, \mathrm{N}-1\}$, measured at $\mathrm{N}$ pixels, may be viewed at a given level of resolution, $\lambda$, to produce an approximation of the signal, denoted $\left\{\mathrm{S}_{\lambda}[\mathrm{n}], \mathrm{n}=0,1, \ldots, \mathrm{N}-1\right\}$. Visually, the resolution may be considered a choice of pixel size for digital representation of a signal, or the smallest selected distance of resolution between two points. A signal may be constructed at a given resolution $\lambda$ by constructing upper and lower bounds to the signal, and then defining $S_{\lambda}$ as an average of the bounds. The bounding signals are designed not only to confine $S$ but also to filter out signal features whose size is less than $\lambda$. Thus, $S_{\lambda}$ also filters out features less than size $\lambda$ and displays behavior at $\lambda$ and larger. This method is meant to emulate the capability of human vision to "broad-brush" a signal in order to filter out unwanted small features. This is shown in Figure 1, which contains a signal viewed in broadbrush or coarse resolution, neglecting smaller visual detail. The coarse or baseline signal follows the trend of the signal and is shown in Figure 2a. Figure $2 \mathrm{~b}$ presents the signal between bounds, with respect to the coarse component, which is noise. Any remaining part of the signal is a peak or trough (with null background), which is presented in Figure 2c. Mathematically this decomposition can be presented as

$$
S_{\text {peaks Irroughs }}(t)=S(t)-S_{\text {baseline }}(t)-S_{\text {noise }}(t)
$$

where $\mathrm{S}(\mathrm{t})$ is the original signal, $\mathrm{S}_{\text {baseline }}(\mathrm{t})$ is the baseline signal, $\mathrm{S}_{\text {noise }}(\mathrm{t})$ is the noise signal and $\mathrm{S}_{\text {peaks/troughs }}(\mathrm{t})$ is the peaks / troughs signal. The decomposition is non-linear (the morphological operations are non-linear), but the components add arithmetically, hence "NLAD" (Non-Linear Addditive Decomposition). The peaks / troughs signal can further be separated into a peaks and a troughs signal.

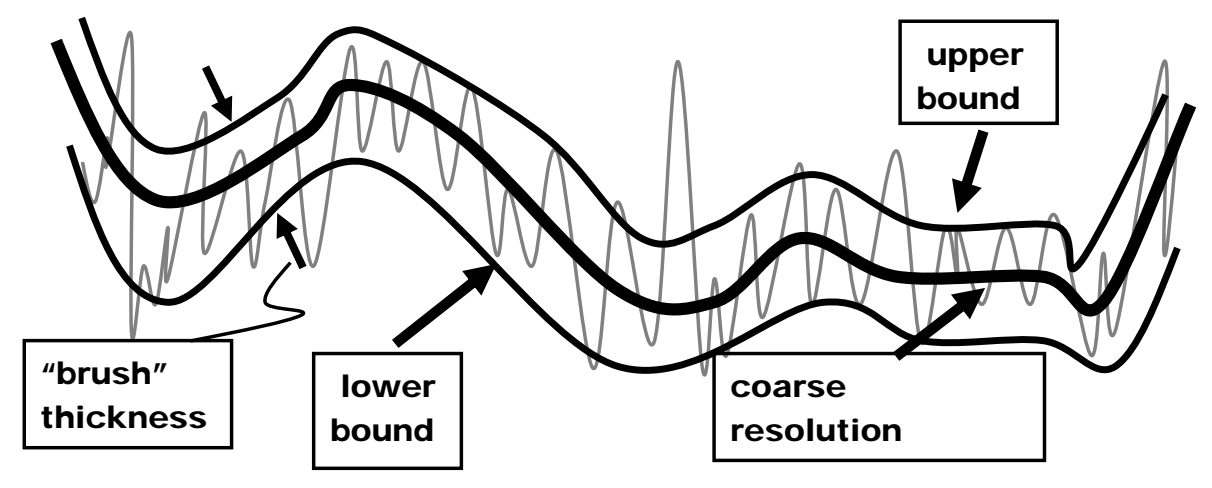

Figure 1. Typical signal

(a)

(b)
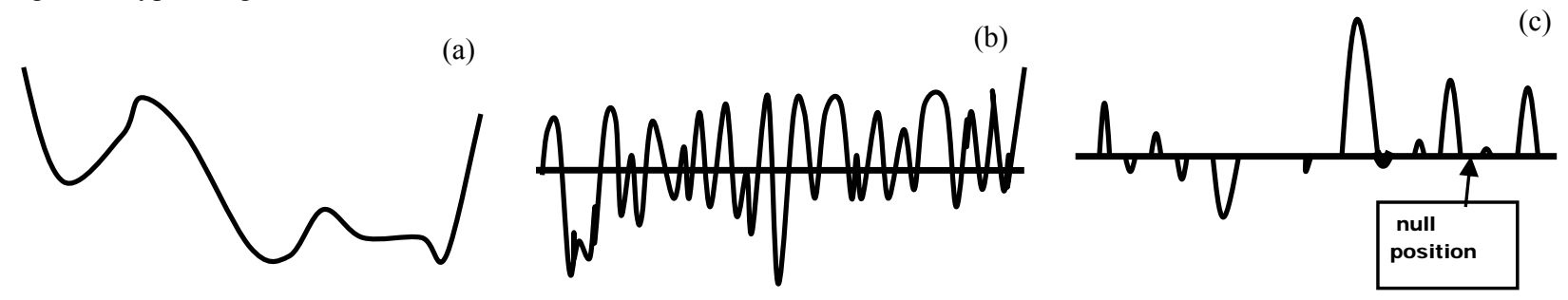

Figure 2. (a) Baseline signal (b) Noise signal (c) Peaks / Trough signal 
The examples presented thus far were one-dimensional signals. NLAD has been extended to two dimensional signals (images). All the features of the 1-D NLAD, discussed above, are also present in the 2-D version. Selection of a resolution level for 1-D NLAD means selection of the length of a line that serves as a structuring element for the performance of mathematical morphology operations on one-dimensional signals. Extension to 2-D NLAD means selection of a region of surface that serves as a structuring element for the performance of mathematical morphology operations on two-dimensional signals. Since it may be assumed that no direction on a surface is different in any way from another direction, the region selected as a structuring element must be spatially symmetric; in other words, the structuring element is a circular disc. The diameter of the disc is interpreted as the resolution distance for the decomposition.

The 2-D NLAD process is described, step-by-step, below. Each step of the formulation will be accompanied by an illustration in order to visualize the operations. The image that will serve as illustration throughout is given in Figure 3a, which is a synthetically constructed gray-scale image. Since it is important to be able to compare gray-scale levels of 2D images, and since such comparisons are often difficult to visualize because the eye cannot distinguish gray-scale levels close in value, a line is drawn over a portion of the image. Figure $3 \mathrm{~b}$ is a graph showing the gray-scale variation of that portion covered by the line (reading left to right). All the processed images derived by NLAD will include a line covering the same pixels as those that appear in Figure 3a. The image will be accompanied by a graph of the gray-scale variation of that portion of the image covered by the line. Gray-scale comparison (on the line) is then easily visualized. A value for resolution level is selected. For the example demonstrated here, the resolution level selected is 19 pixels (i.e., the structuring element is a disc of diameter 19 pixels).

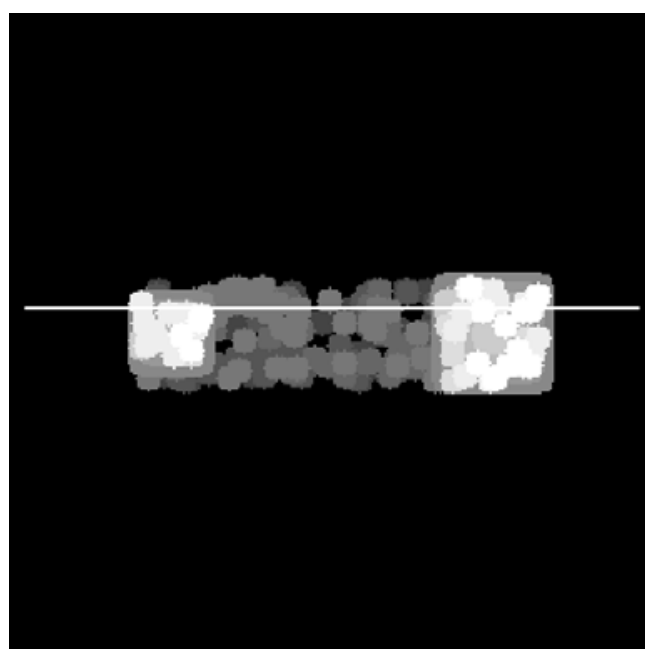

(a)

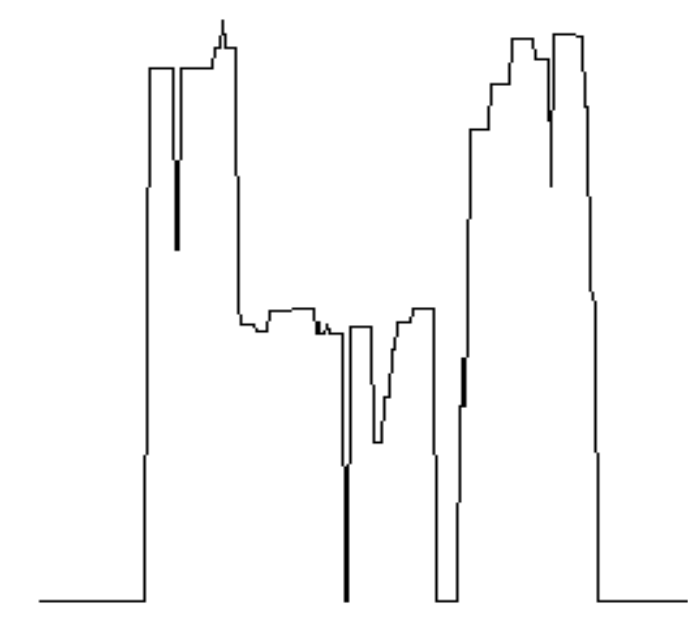

(b)

Figure 3. (a) Gray-scale image with line on image and (b) Pixel gray-scale values on line.

\subsection{Boundary surfaces}

Two gray-scale "boundary" surfaces are constructed, one that bounds the image from above ("above", meaning in grayscale value) and one that bounds the image from below. The upper boundary does not bound the image from above everywhere, nor does the lower boundary bound the image from below everywhere. Distances are viewed at the assigned resolution level, so that functional behavior that is finer than that at the chosen resolution level is smoothed. This means that peaks and troughs whose widths are narrower than the resolution value are clipped off, i.e., they reside outside the boundaries established by the upper and lower boundary surfaces. Boundary surfaces are constructed in the following manner.

Morphological Close Operation:

1. Perform $D(I, s e)[p]=M A X[I(p-z)]$, $z \in S e$ 
where " $D$ " stands for the morphological Dilate operator, $I$ represents the image, and "se" stands for the structuring element (a disc). Thus the value at pixel "p" is the maximum pixel value of $I$ among all locations that are covered by a disc whose origin lies on pixel "p".

2. Perform $C(I, s e)[p] \equiv E(D(I, s e), s e)[p]=\operatorname{MIN}[D(p+z)]$,

$$
z \in \text { se }
$$

where " $C$ " stands for the morphological Close operator and " $E$ " stands for the morphological Erode operator. The value of Erode at pixel "p" is the minimum pixel value of $D(I, s e)$ among all locations covered by a disc whose origin lies on pixel "p". The combination of Dilate followed by Erode is called a Close morphological operation.

Morphological Open Operation:

1. Perform $E(I, s e)[p]=\operatorname{MIN}[I(p+z)]$,

$$
z \in s e
$$

2. Perform $O(I, s e)[p] \equiv D(E(I, s e), s e)[p]=\operatorname{MAX}[E(p-z)]$,

$$
z \in \text { se }
$$

where " $O$ " represents the morphological Open operator. The combination of Erode followed by Dilate is called an Open morphological operation.
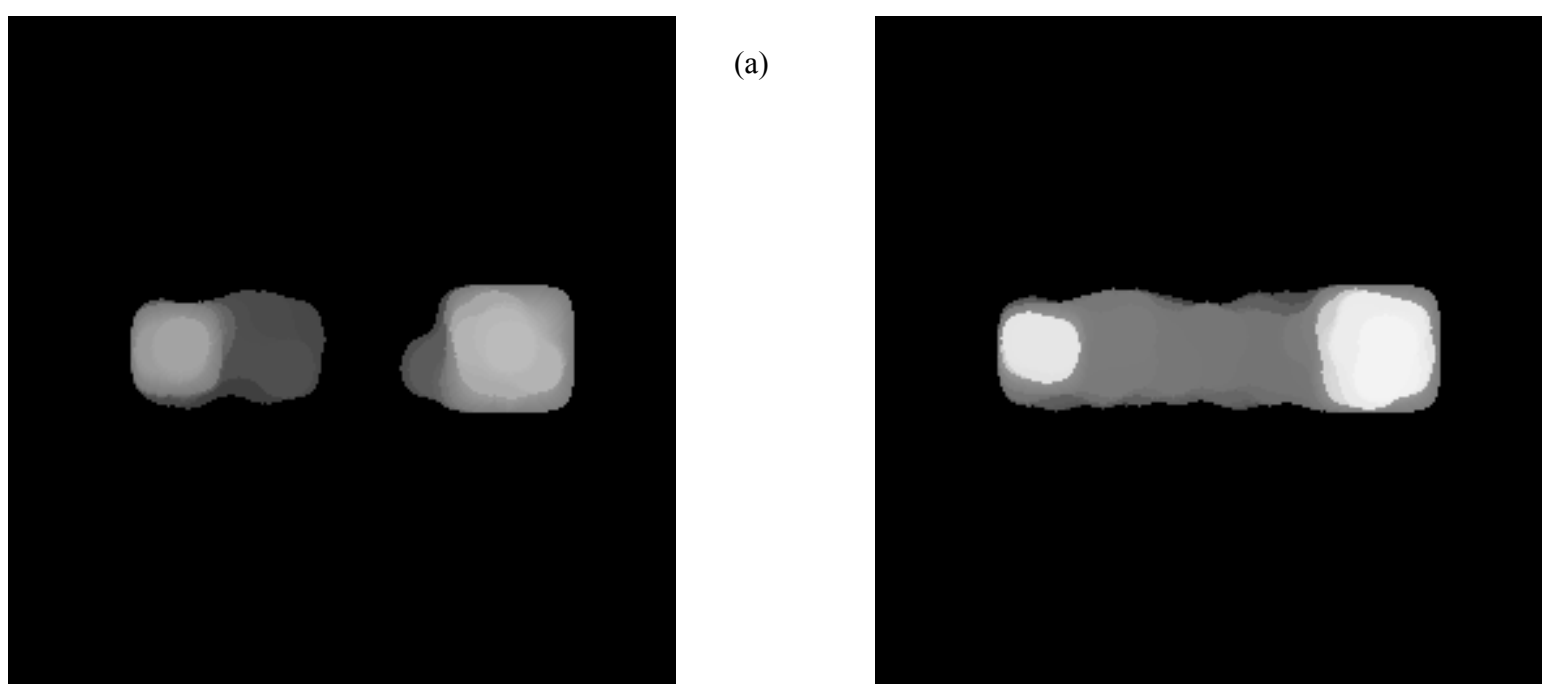

Figure 4. (a) Lower bound surface and (b) upper bound surface of Figure 3a

Upper boundary surface:

UpperBnd $(I, s e)[p]=O(C(I, s e), s e)[p]$,

i.e., first perform a morphological Close on the image $I$, followed by a morphological Open on the Close surface.

Lower boundary surface:

LowerBnd $(I, s e)[p]=C(O(I, s e), s e)[p]$,

i.e., first perform a morphological Open on the image $I$, followed by a morphological Close on the Open surface. Figure 4 shows the lower and upper bounds of the image. 


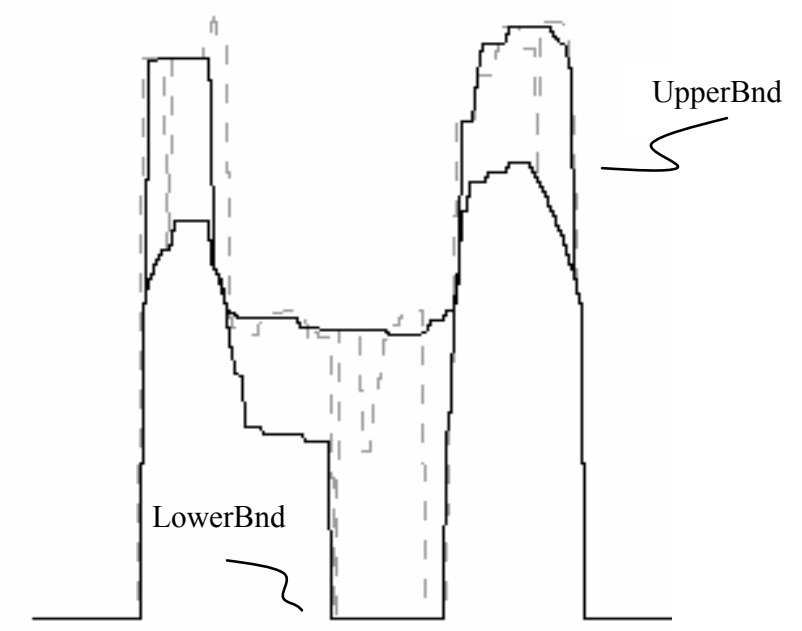

Figure 5. Gray-scale values of the lower and upper bound surfaces (full lines) along the line of Figure 3a. The gray-scale values of the image along the line (Figure 4) are included for comparison (broken line).

Figure 5 is a graph of the lower and upper bound surfaces along the line shown in Figure 3a. The condition LowerBnd[p] $\leq$ UpperBnd [p], as defined above, is true almost everywhere, although, under certain image conditions, this inequality does not hold. It is important in the definition of the baseline component that the inequality holds everywhere, so that LowerBnd $[p]$ and UpperBnd $[p]$ are modified by

$$
\begin{aligned}
& \text { LowerBnd }[p] \Rightarrow \operatorname{MIN}(\text { LowerBnd,UpperBnd })[p]) \equiv \operatorname{LowerBnd}[p] \text { and } \\
& \text { UpperBnd }[p] \Rightarrow \operatorname{MAX}(\text { LowerBnd,UpperBnd })[p]) \equiv \operatorname{UpperBnd}[p] .
\end{aligned}
$$

Also note that these two "boundary" surfaces do not bracket the image $I[p]$ everywhere. As mentioned earlier, this lack of total bracketing is due to the resolution-based approximation of the image boundaries.

\subsection{Baseline Component Surface}

The lower and upper bound surfaces display the minimum and maximum behavior of the gray-scale surface, image $I$, at the selected resolution level. The trend, or baseline, component may be defined as the average of these minimum and maximum resolved surfaces. Accordingly,

$$
\text { BaselineComponent }[p] \equiv 1 / 2(\text { UpperBnd }[p]+\text { LowerBnd }[p]) \text {. }
$$

Figure $6 \mathrm{a}$ shows the baseline component of the image. Figure $6 \mathrm{~b}$ is a graph showing the gray-scale values corresponding to the reference line; the upper and lower bound surfaces along the line are included for comparison. Note that

\section{LowerBnd $[p] \leq$ BaselineComponent $[p] \leq$ UpperBnd $[p]$ everywhere.}

\subsection{Noise Component Surface}

The noise component represents gray-scale value detail at resolution levels finer (less) than that selected for image decomposition. It is represented by the behavior of the gray-scale surface within the upper and lower bounds, with respect to the baseline. Thus, the Noise Component Surface may be defined as follows. 


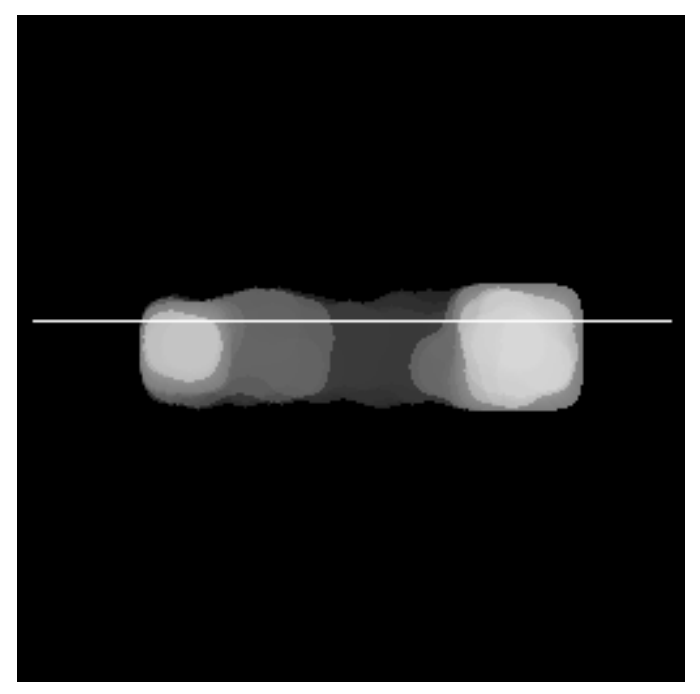

(a)

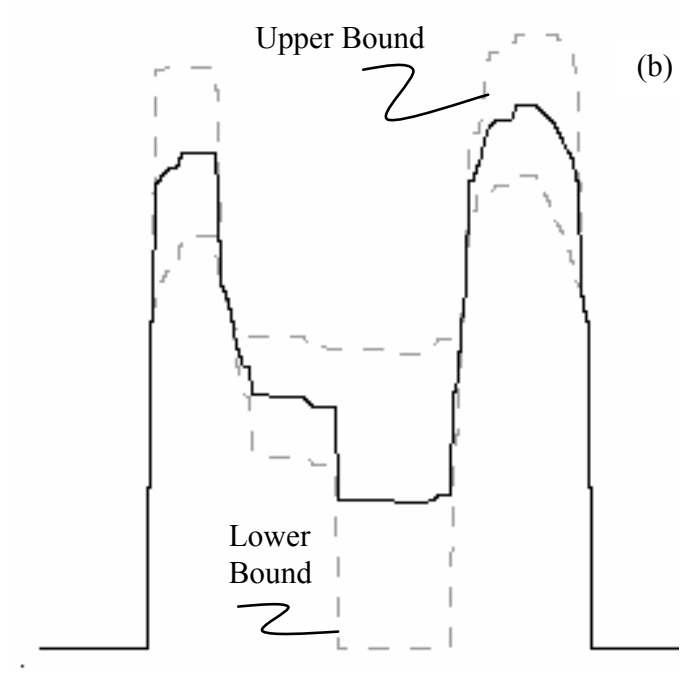

Figure 6. (a) Baseline component with line. (b) Baseline component on line (full line), bracketed by Low- and Upper bounds on line (broken lines).

$$
\begin{array}{rlrl}
\text { NoiseComponent }[p]=I[p]-\text { BaselineComponent }[p], & & \text { LowerBnd }[p] \leq I[p] \leq \text { UpperBnd }[p] \\
& =\text { UpperBnd }[p]-\text { BaselineComponent }[p], & & \text { UpperBnd }[p] \leq I[p] \\
& =\text { LowerBnd }[p]-\text { BaselineComponent }[p], & I[p] \leq \operatorname{LowerBnd}[p]
\end{array}
$$

For the example image, the Noise Component Surface is shown in Figure 7a. The gray-scale values of the Noise Component on the line are shown in Figure $7 \mathrm{~b}$.

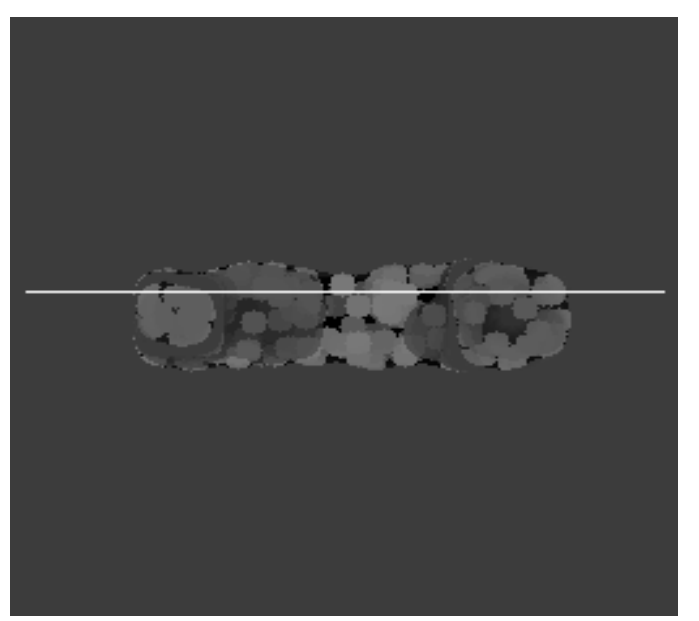

(a)

Figure 7. (a) Noise component surface with line. (b) Noise component values on line.

\subsection{Peaks and Troughs Component Surfaces}

The peaks component surface is defined in the peaks region, UpperBnd $[p] \leq I[p]$, whereas the troughs component surface is defined in the troughs region, $I[p] \leq \operatorname{LowerBnd}[p]$, that is, wherever the gray-scale surface lies outside of the UpperBnd or LowerBnd surfaces respectively. Thus, 

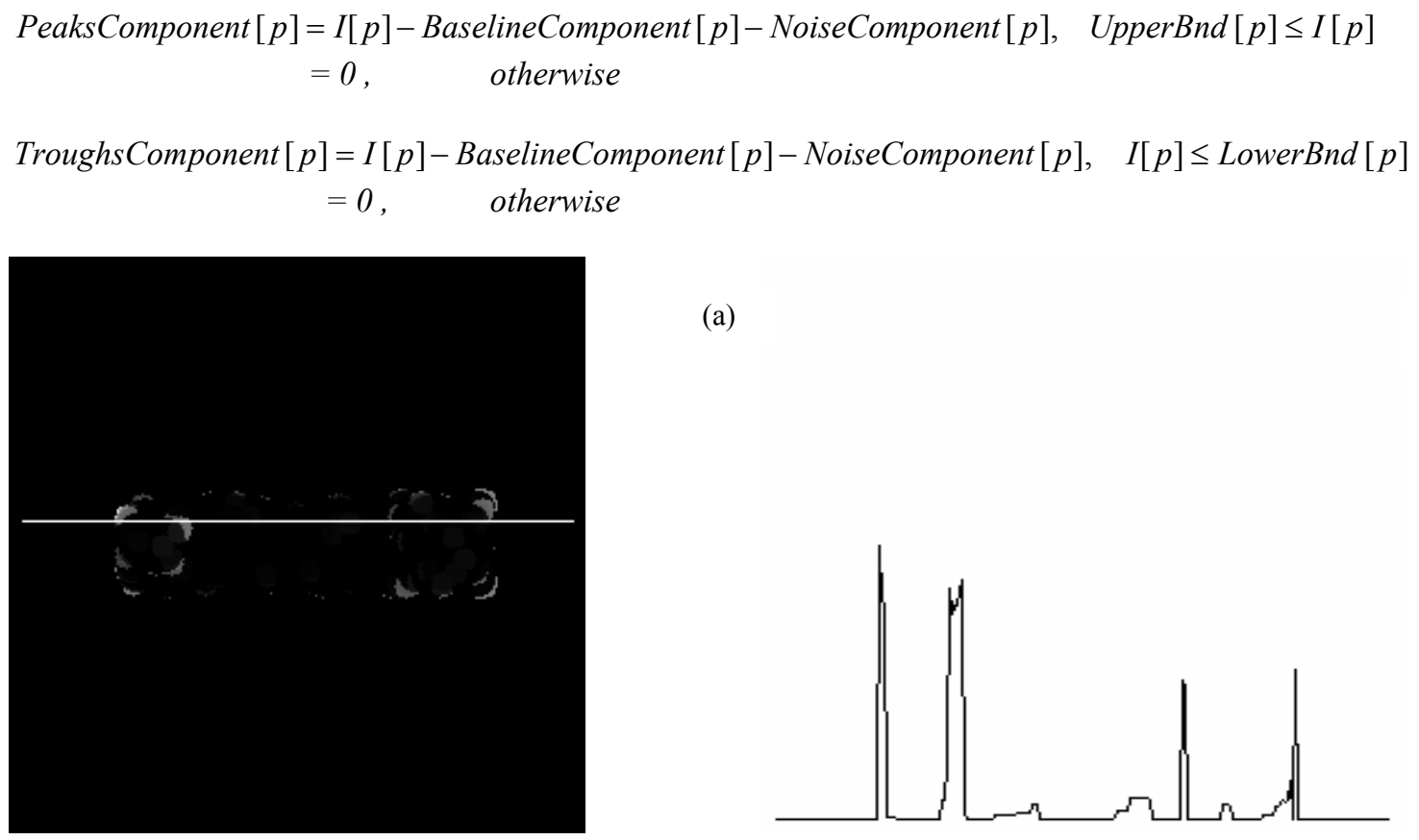

(a) (b)

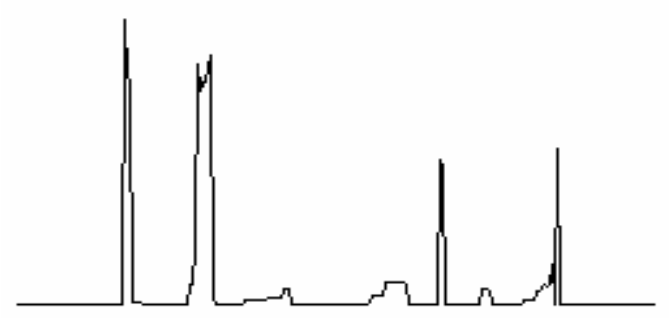

Figure 8. (a) Peaks Component surface of Figure 3a image with line. (b) Gray-scale values of peaks on line.

The peaks or troughs are prominent features of the respective components because they are defined with respect to a zero base. For the example, Figure 3a image, the Peaks Component Surface is shown in Figure 8a. The values of the Peaks Component on this line are shown in Figure 8b. Similar illustrations for the Troughs Component appear in Figure 9. Because all pixel values have been defined to be positive, the values of the Troughs Component actually represent the absolute values of that component. In this example the heights of the peaks and depths of the troughs in their respective regions are not large. Indeed it is not possible for the eye to distinguish the small non-zero values within the trough region from the background zero values.

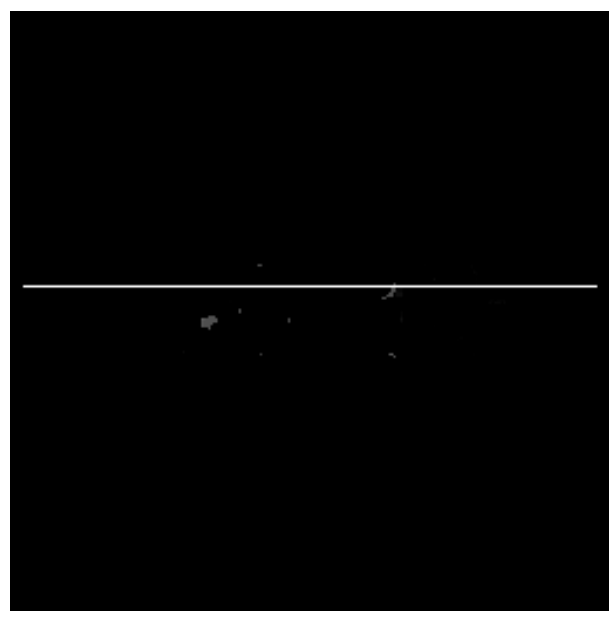

Figure 9. (a) Absolute value of the Trough Component surface of Figure 3a image with line. (b) Absolute values of the Trough Component on the line.

The morphological operations used to perform the decomposition are manifestly non-linear. However, by definition, the decomposition is additive everywhere: 


\section{$I[p]=$ BaselineComponent $[p]+$ NoiseComponent $[p]+($ PeaksComponent $[p]+$ TroughsComponent $[p])$}

Consequently, all information concerning the image may be retained after decomposition is performed; nothing is lost.

The decomposition is also clean, in the sense that the information contained in one component does not appear in another component. For example, the noise component is not contaminated by coarse resolution trends across the image, nor does it contain peaks or troughs at the given resolution level. Similarly, the baseline component contains no noise or no peaks/troughs information, and likewise for the peaks and troughs components. As a result, information of one type can be removed from an image by arithmetic subtraction without affecting information of another type. For example, noise of small amplitude can be removed from an image without removing trend or prominent features of a certain size, thereby providing a sharper image. Another example is an image that contains spurious small patches of high intensity. The small high intensity patches, considered as gray-scale peaks, may be isolated in the peaks component of the image, by performing NLAD at an appropriate resolution level, and then subtracting the peaks component from the image. Another example is identifying a coarse resolution trend in an image, caused by illumination variations, as constituting the baseline component of the NLAD of the image. Removing the baseline component by subtraction simulates evenly distributed lighting conditions.

NLAD has several additional advantages over conventional image processing algorithms, including no need for a transformation from one space to another, such as is done with Fourier transforms, and since only finite summations are required, the calculational effort is neither extensive nor complicated. There is also no need for selecting a basis function as is done for wavelet analysis.

Another important feature of the NLAD algorithm is that it can detect the peaks correctly in an image where there is a background intensity gradient, while other techniques such as thresholding cannot. Figure 10 is a representation of the line profile of the gray-scale intensity across an image which has a non-uniform background intensity. Peak " $\mathrm{A}$ " is included when the image is thresholded at the threshold value shown, but all other peaks are excluded. Some excluded peaks may have high gray-scale values (such as peaks "B" and "C"). Any other threshold value that includes all peaks includes non-peak sections, as well. In general, there is no way to know whether it is possible to find image threshold values that will include all peaks and only peaks. As a consequence, it is also not possible to know with confidence whether simple gray-scale thresholding can give a true quantitative representation of the peaks of an image. In contrast, NLAD can detect the peaks with ease.

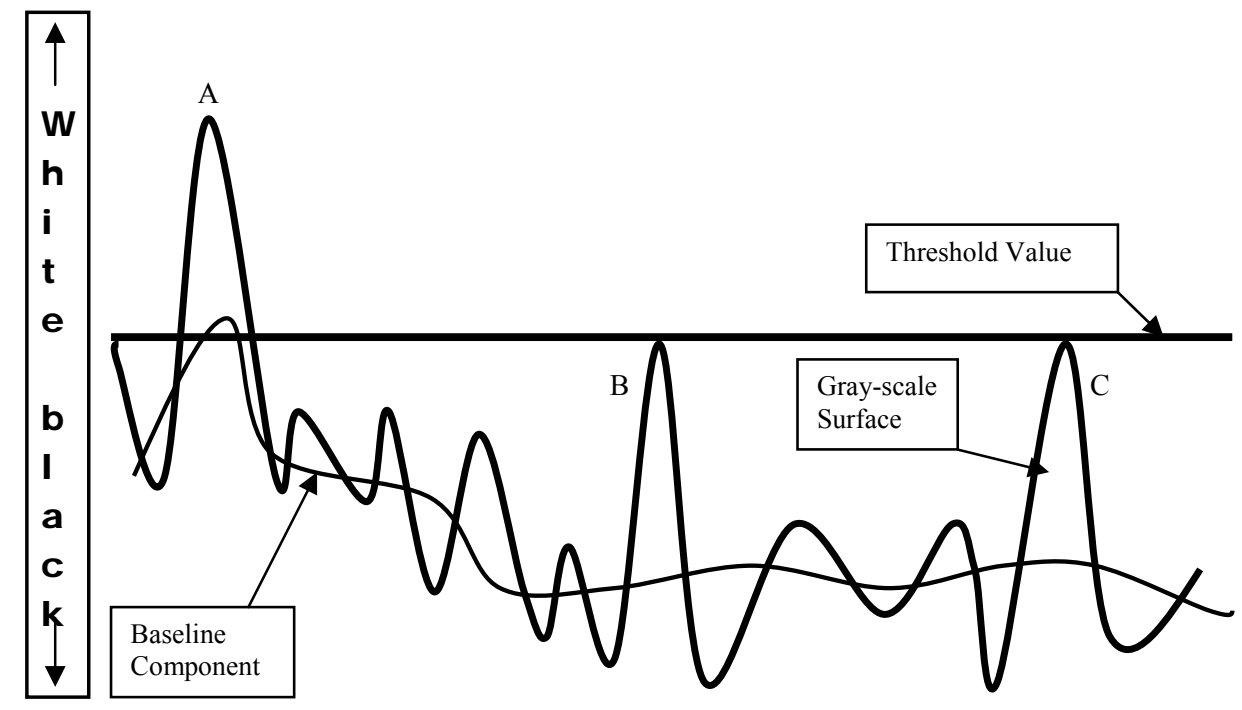

Figure 10. Line Profile of the Gray-scale Intensity Across an Image 


\section{APPLICATION OF 2-D NLAD}

A parametric study was performed to determine the detection capability of the 2-D NLAD algorithm. Images containing circles, ovals and rectangles of different sizes and gray-scale intensities were analyzed at several NLAD resolutions. The gray-scale intensity of these objects is different than the surrounding image background. The range of sizes examined was arbitrarily selected to demonstrate the detection capability and not to define the size limit of detection. The peaks component image is used as the result in this section.

Figure 11a shows the first test image. This image contains five rows of circles with diameters ranging from 4 pixels to 40 pixels. Random noise (with an intensity level between 0 and 9) was added to the image. The relative gray-scale intensity above the average background noise for each circle is 15 . The relative gray-scale intensity is calculated by subtracting the root mean square (rms) value of the background noise for the image from the absolute gray-scale intensity. An intensity gradient (across the image width) with a slope of 0.1 was also added to the image. A line profile of the gray-scale intensity across the image width is presented in Figure 11b. The intensity gradient can be clearly seen in this figure. This is to demonstrate NLAD's capability of detecting objects in an image with a non-uniform background intensity.

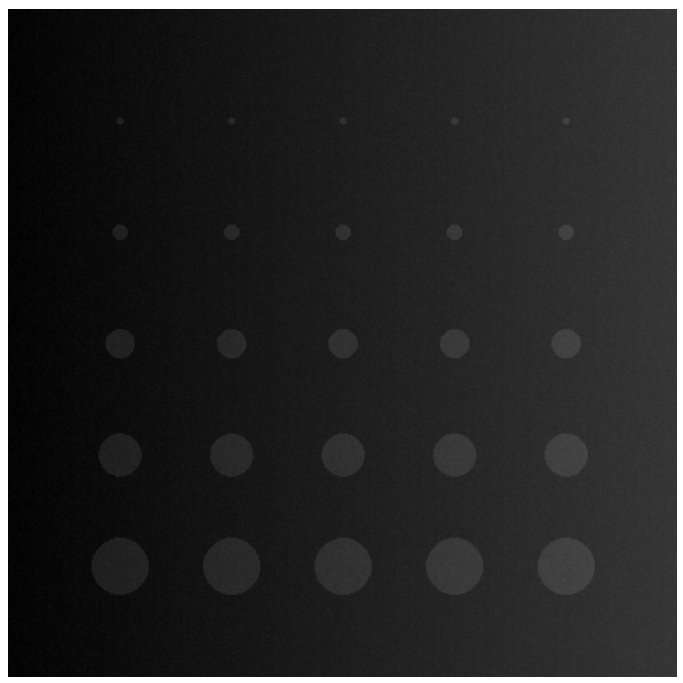

(a)

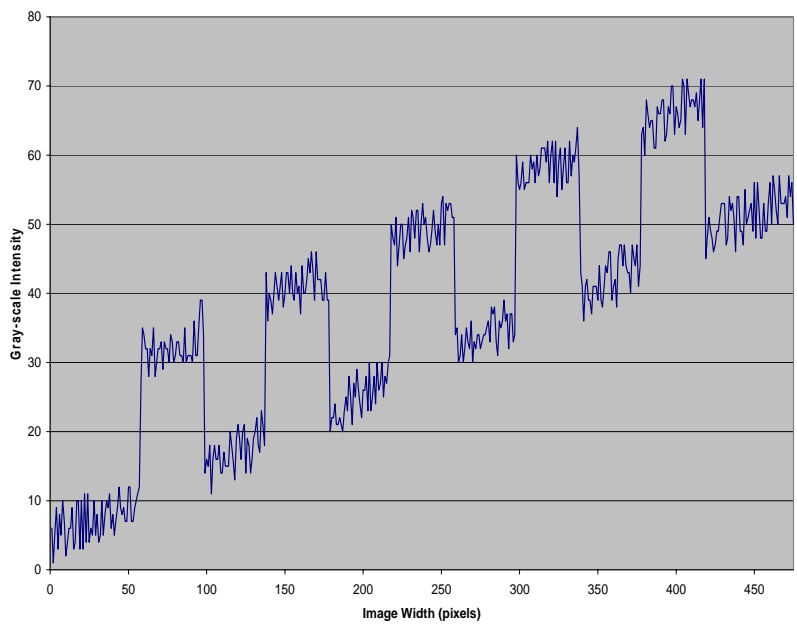

Figure 11 (a) Circles Test Image (b) Line Profile of Gray-scale Intensity Across Image Width for Circles Test Image

The image was analyzed using the NLAD algorithm. The resolution was varied between 10 and 100 to examine the effect of varying the resolution. Figure 12 shows the results of this analysis. The percent of total area detection is defined as

$$
\% \text { Total Area }=\frac{\text { Area of Objects Detected by NLAD }}{\text { Actual Area of Objects }} \times 100 \%
$$

The optimal resolution is defined as the resolution at which all the objects in the image are detected completely (\%Total Area $=100 \%$ ). For the images in this paper, there is a range of resolutions at which all objects are detected. This is called the optimal resolution range. At resolutions smaller than the optimal resolution range (e.g., diameter $=20$ ), the area detected by the algorithm is less than the actual area of the circles in Figure 11a. At resolutions much larger than the objects in the image (e.g., diameter $=90$ ), the detected area is also much less than the actual area. But at the intermediate resolution levels (diameter $=40$ to 70$), 100 \%$ of the actual area of the objects are detected by the algorithm. The reason for this is as follows. When the resolution is less than the optimal resolution range, ("fine" level of resolution, diameter = 20 pixels), i.e., at levels that are smaller than those of the object sizes in this image, parts of the larger objects are 
included as part of the baseline (trend) image. Thus the peaks image total area is smaller than the actual peaks total area. On the other hand, when the resolution is greater than the optimal resolution range, ("coarse" level of resolution, diameter $=90$ pixels), i.e., at values of resolution that are significantly greater than the sizes of the majority of the objects in this image, portions of the smaller objects become hidden in the image noise. Thus the peaks image total area is smaller than the actual peaks total area. But when the resolution is in the optimal resolution range all the objects are detected. This demonstrates that the algorithm is able to accurately detect the objects which have different intensities than the surrounding image background when there is a non-uniform background intensity.

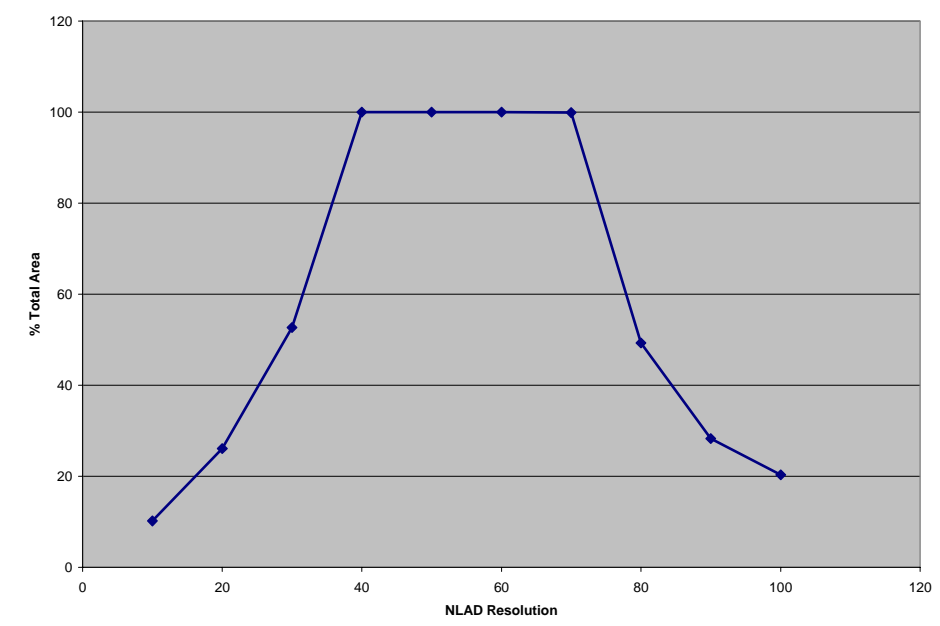

Figure 12. Comparison of \% Total Area for Circles Test Image for Various NLAD Resolutions

The optimal resolution for any image is dependent on the size of the objects and the relative spacing between the objects. When the spacing between objects is larger than the resolution value there is no dependency. On the other hand, when the spacing between objects is less than the resolution, portions of the objects are captured in both the trend and noise and the area detected can be less than the actual area. This is because during the decomposition, objects in close proximity to each other (relative to the resolution value) are not distinguished as individual objects and are identified as a single object. This is shown in Figure 13a, which is a plot of the maximum percent total area detected as a function of the distance between the circle edges. Six test images with a range of spacing between the circles were analyzed using NLAD. Each test image contained circles of the same size and intensity that were equidistant from each other. The distance between the circles for the images varied from $0.25 \mathrm{D}$ to $2.5 \mathrm{D}$ where $\mathrm{D}$ is the diameter of circles. Each point on the graph is the maximum percent total area detected for an image. An example of one of these images is shown in Figure 13b. This image contains circles with the distance between the circle edges equal to the diameter of the circles. The maximum total area for each image is obtained by varying the resolution as discussed above. One can see from this figure, as long as the distance between the edge of an object is greater than the diameter of the objects, the NLAD algorithm accurately detects the actual area of the objects in the image. On the other hand, if the distance between the objects is less than the diameter of the objects, the maximum area detected by the algorithm is less than the actual area of the objects in the image.

Test images containing ellipses and rectangles, presented in Figures 14a and 15a, respectively, were also analyzed using the 2-D NLAD algorithm. The ovals ranged in size from 8 pixels $x 4$ pixels (major axis x minor axis) to 56 pixels $\mathrm{x} 28$ pixels, while the rectangles length $\mathrm{x}$ width are the same size as the major and minor axes of the ellipses. As with the circle test image, the relative intensity of each oval and rectangle is 15 and an intensity gradient across the image width was also added to the images. The sizes were chosen to match the area of the circles in Figure 5 as close as possible, while maintaining an aspect ratio of 2 to 1 for each ellipse and a length to width ratio of 2 to 1 for the rectangles. The results of analyzing these images with resolutions between 10 and 100 for the ellipses and rectangles are shown in Figures $14 \mathrm{~b}$ and $15 \mathrm{~b}$, respectively. The same trend as with the circles is also present, the area detected by the algorithm is less than the actual area when the resolution is less than or greater than the optimal resolution range. The optimal resolution range occurs between 30 and 70 for the ellipses and between 30 and 60 for the rectangles. 


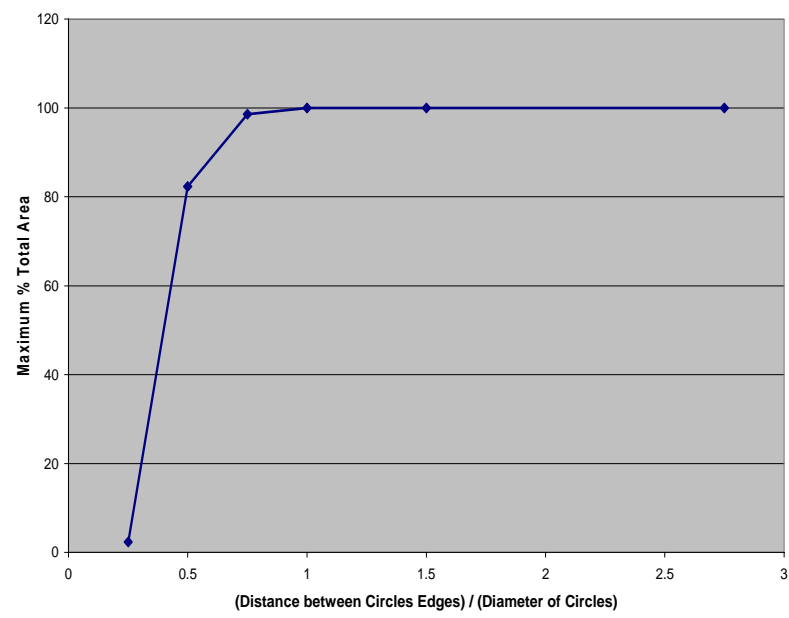

(a)

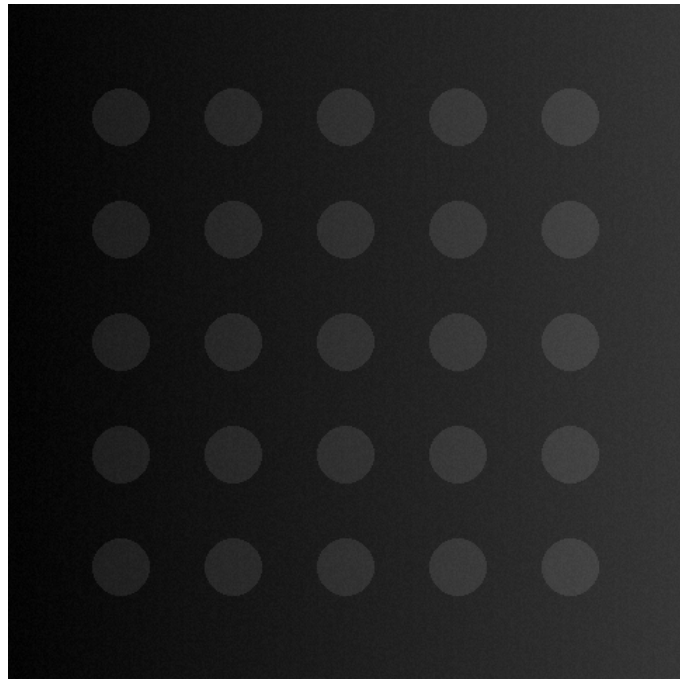

(b)

Figure 13 (a) Maximum Percent Total Area Detected as a Function of Distance Between Edges of the Circles (b) Circles Test Image: Distance Between Circle Edges $=$ Circle Diameter

(a)

(b)
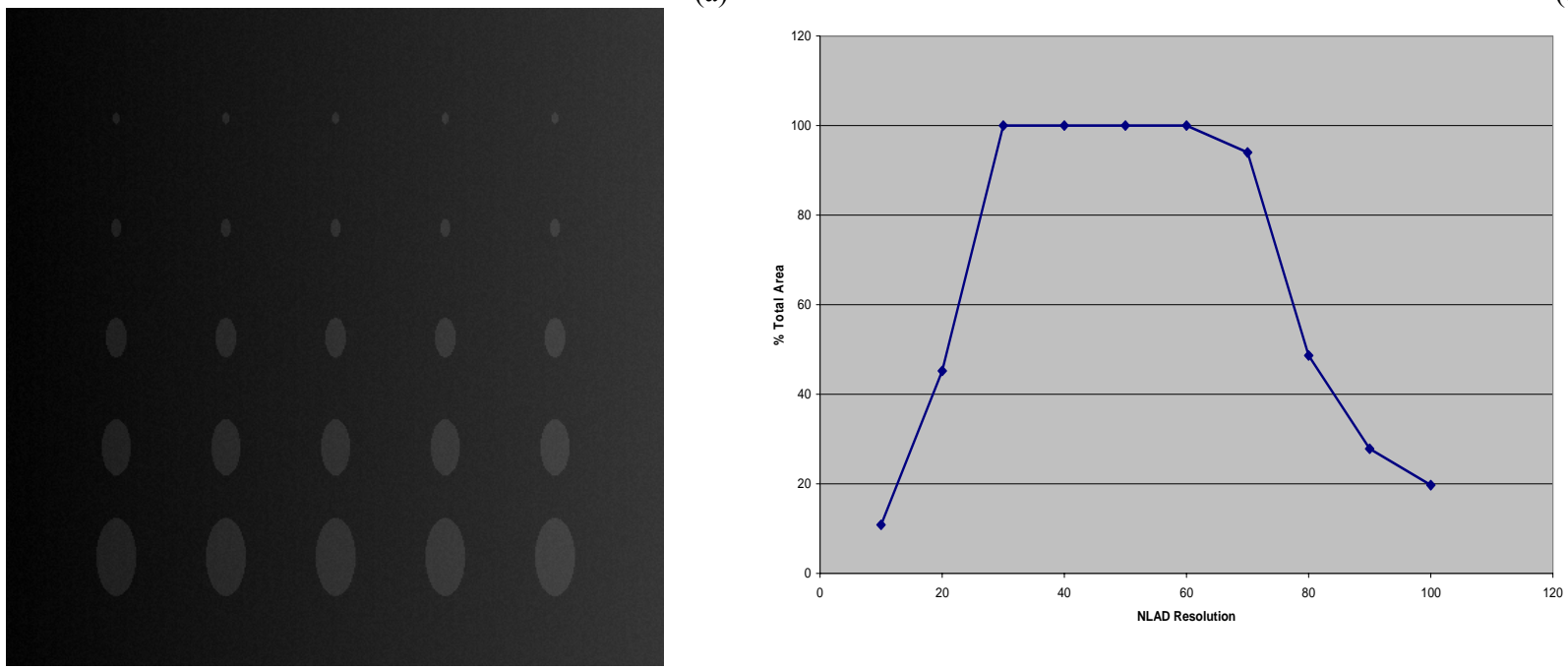

Figure 14 (a) Ovals with Intensity Gradient Across Image Width Test Image (b) Comparison of \% Total Area of Ovals for Various NLAD Resolutions

Thus to detect the actual area of objects with varying sizes and shapes, the algorithm should be run over a range of resolution values. This range of resolutions should cover the range of sizes of the objects being considered for detection. The resolutions should range from smaller than the size of the smallest objects to larger than the size of the largest objects in the image. The resolution at which the maximum area is detected is usually the optimal resolution that will give the true area of the bright regions in the image. One needs to examine the objects selected by the algorithm to make sure they are correct. Typically, the optimal resolution is at or slightly larger than the largest object in the image. 


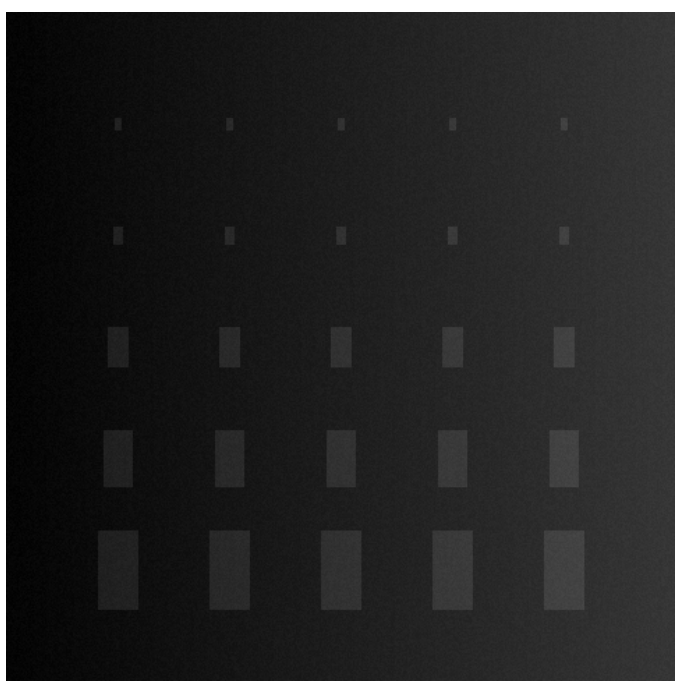

(a)

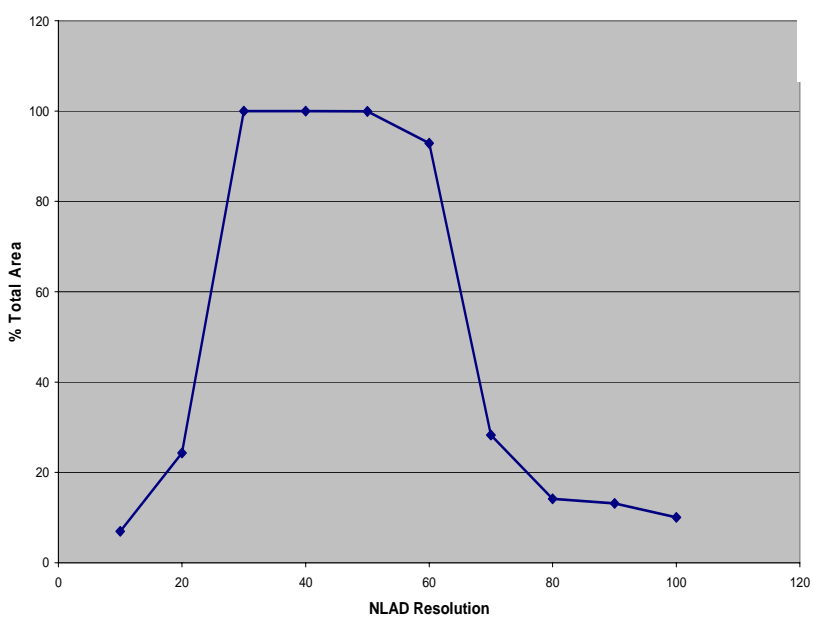

(b)

Figure 15 (a) Rectangles Test Image (b) Comparison of Total Area of Rectangles for Various NLAD Resolutions

\section{CONCLUSIONS}

A new image processing algorithm, two-dimensional non-linear additive decomposition (NLAD), which is used to identify regions in a digital image whose gray-scale (or color) intensity is different than the surrounding background, is presented in this paper. The decomposition techniques used in this algorithm are based on the concepts of mathematical morphology. NLAD emulates the human capability of visually separating an image into different levels of resolution, denoted as 'coarse', 'fine', and 'intermediate.' The decomposition is performed in such a way that very little resolution information overlaps any two of the components. NLAD can easily remove noise and background intensity variations from an image. Some additional advantages of NLAD over conventional image processing algorithms are that the calculational effort is neither difficult nor extensive because only finite summations are required and that no transformations from one space to another are required. There is also no need for selecting a basis function as is done for wavelet analysis.

\section{REFERENCES}

1. J. Serra, Image Analysis and Mathematical Morphology, Vol. 1, 1982.

2. G. Matheron, Elements pour une Theorie des Milieux Poreux, 1967.

3. J. Serra, Mathematical Morphology, Vol. 2, 1988.

4. R.M. Haralick, S.R. Sternberg, and X. Zhuang, Image Analysis using Mathematical Morphology, "IEEE Transactions on Pattern Analysis and Machine Intelligence,” Vol. PAMI-9, No. 4, pp. 532-550, July 1987.

5. M. Sonka, V. Hlavac, R. Boyle, Image Processing Analysis and Machine Vision, PWS Publishing, 1999.

6. D. Brudnoy, Method and Apparatus for Automatically Detecting Pattern in Digital Point-Ordered Signals, U.S. Patent Number 5,825,672, 1996. 